\title{
From the Bose-Einstein to Fermion Condensation
}

\author{
M.Ya. Amusia ${ }^{a, b}$, A.Z. Msezane ${ }^{c}$, and V.R. Shaginyan ${ }^{c, d *}$ \\ ${ }^{a}$ The Racah Institute of Physics, the Hebrew University, Jerusalem 91904, Israel; \\ ${ }^{b}$ A. F. Ioffe Physical-Technical Institute, 194021 St. Petersburg, Russia; \\ ${ }^{c}$ CTSPS, Clark Atlanta University, Atlanta, Georgia 30314, USA; \\ ${ }^{d}$ Petersburg Nuclear Physics Institute, RAS, Gatchina, 188300, Russia
}

\begin{abstract}
The appearance of the fermion condensation, which can be compared to the BoseEinstein condensation, in different liquids is considered, its properties are discussed, and a large number of experimental evidences in favor of the existence of the fermion condensate (FC) is presented. We show that the appearance of FC is a signature of the fermion condensation quantum phase transition (FCQPT), that separates the regions of normal and strongly correlated electron liquids. Beyond the FCQPT point the quasiparticle system is divided into two subsystems, one containing normal quasiparticles and the other - FC localized at the Fermi level. In the superconducting state the quasiparticle dispersion in systems with FC can be represented by two straight lines, characterized by effective masses $M_{F C}^{*}$ and $M_{L}^{*}$, and intersecting near the binding energy $E_{0}$, which is of the order of the superconducting gap. The same quasiparticle picture and the energy scale $E_{0}$ persist in the normal state. We demonstrate that fermion systems with FC have features of a "quantum protectorate" and show that strongly correlated electron systems with FC, which exhibit large deviations from the Landau Fermi liquid behavior, can be driven into the Landau Fermi liquid by applying a small magnetic field $B$ at low temperatures. Thus, the essence of strongly correlated electron liquids can be controlled by weak magnetic fields. A re-entrance into the strongly correlated regime is observed if the magnetic field $B$ decreases to zero, while the effective mass $M^{*}$ diverges as $M^{*} \propto 1 / \sqrt{B}$. The regime is restored at some temperature $T^{*} \propto \sqrt{B}$. The behavior of Fermi systems which approach FCQPT from the disordered phase is considered. This behavior can be viewed as a highly correlated one, because the effective mass is large and strongly depends on the density. We expect that FCQPT takes place in trapped Fermi gases and in a low density neutron matter leading to stabilization of the matter by lowering its ground state energy. When the system recedes from FCQPT the effective mass becomes approximately constant and the system is suited perfectly to be conventional Landau Fermi liquid.
\end{abstract}

PACS numbers: 71.27.+a, 74.20.Fg, 74.25.Jb

Typeset using REVTEX

*E-mail: vrshag@thd.pnpi.spb.ru 


\section{INTRODUCTION}

Experimental and theoretical explorations of Bose systems below the temperatures of Bose-Einstein condensation have entailed great difficulties. Among the pioneers of the theoretical studies is S.T. Belyaev ${ }^{1}$ in whose papers a solid base for taking into account the interaction among Bosons at low temperatures has been established $[1,2]$.

In a system of interacting bosons at temperatures lower than the temperature of Bose-Einstein condensation, a finite number of particles is concentrated in the lowest level. In case of a noninteracting Bose gas at the zero temperature, $T=0$, this number is simply equal to the total number of particles in the system. In a homogeneous system of noninteracting Bosons, the lowest level is the state with zero momentum, and the ground state energy is equal to zero. For a noninteracting Fermi system such a state is impossible, and its ground state energy $E_{g s}$ reduces to the kinetic energy and is proportional to the total number of particles. Imagine an interacting system of fermions with a pure repulsive interaction. Let us increase its interaction strength. As soon as it becomes sufficiently large and the potential energy starts to prevail over the kinetic energy, we can expect the system to undergo a phase transition when a finite number of the Cooper like pairs with an infinitely small binding energy can condensate at the Fermi level. Such a state resembles the Bose-Einstein condensation and can be viewed as fermion condensation. This phase transition leads to the onset of the fermion condensate (FC) and separates a strongly interacting Fermi liquid from a strongly correlated one. Lowering the potential energy, the fermion condensation decreases the total energy. Unlike the Bose-Einstein condensation, which occurs even in a system of noninteracting bosons, the fermion condensation can take place if the coupling constant of the interaction is large, or the corresponding Landau amplitudes are large and repulsive.

One of the most challenging problems of modern physics is the structure and properties of systems with large coupling constants. It is well-known that a theory of liquids with strong interaction is close to the problem of systems with a big coupling constant. The first solution to this problem was offered by the Landau theory of Fermi liquids, later called "normal", by introducing the notion of quasiparticles and parameters, which characterize the effective interaction among them [3]. The Landau theory can be viewed as the low energy effective theory in which high energy degrees of freedom are removed at the cost of introducing the effective interaction parameters. Usually, it is assumed that the stability of the ground state of a Landau liquid is determined by the Pomeranchuk stability conditions: the stability is violated when even one of the Landau effective interaction parameters is negative and reaches a critical value. Note that the new phase, new ground state, at which the stability conditions are restored can in principle be again described within the framework of the same theory.

It has been demonstrated, however rather recently [4] that the Pomeranchuk conditions cover not all possible instabilities: one of them is missed. It corresponds to the situation when, at the temperature $T=0$, the effective mass, the most important characteristic of Landau quasiparticles,

\footnotetext{
${ }^{1}$ This paper is dedicated to the eightieth birthday of S.T. Belyaev, whose contribution to the modern theoretical physics is enormous indeed. His interest in and deep understanding of different domains of physics, including the experimental one, is very impressive. Always on the front line of scientific research, he is a source of inspiration for mature physicists and an excellent role model for the beginners. We sincerely wish S. T. Belyaev long healthy years to come.
} 
can become infinitely large. Such a situation, leading to profound consequences, can take place when the corresponding Landau amplitude being repulsive reaches some critical value. This leads to a completely new class of strongly correlated Fermi liquids with FC [4,5], which is separated from that of a normal Fermi liquid by the fermion condensation quantum phase transition (FCQPT) [6].

In the FCQPT case we are dealing with the strong coupling limit where an absolutely reliable answer cannot be given on the bases of pure theoretical first principle foundation. Therefore, the only way to verify that FC occurs is to consider experimental facts, which can be interpreted as confirming the existence of such a state. We believe that these facts are seen in some features of those two-dimensional (2D) systems with interacting electrons or holes, which can be represented by doped quantum wells and high- $T_{c}$ superconductors. Considering the heavy-fermion metals, the $2 \mathrm{D}$ systems of ${ }^{3} \mathrm{He}$, the trapped neutrons and Fermi gases, we will show that FC can exist also in these systems.

The goal of our paper is to describe the behavior of Fermi systems with FC and to show that the existing data on strongly correlated liquids can be well understood within the theory of Fermi liquids with FC. In Sec. II, we review the general features of Fermi liquids with FC in their normal state. Sec. III is devoted to consideration of the superconductivity in the presence of FC. We show that the superconducting state is totally transformed by the presence of FC. For instance, the maximum value $\Delta_{1}$ of the superconducting gap can be as large as $\Delta_{1} \sim 0.1 \varepsilon_{F}$, while for normal superconductors one has $\Delta_{1} \sim 10^{-3} \varepsilon_{F}$. Here $\varepsilon_{F}$ is the Fermi level. In Sec. IV we describe the quasiparticle's dispersion and its lineshape and show that they strongly deviate from the case of normal Landau liquids. In Sec. V we apply our theory to explain the main properties of heavy-fermion metals. We demonstrate that it is possible to control the main properties, or even the essence, of strongly correlated electron liquids by weak magnetic fields. Sec. VI deals with the possibility of FCQPT in different Fermi systems, such as $2 \mathrm{D}$ systems of electrons and $2 \mathrm{D}{ }^{3} \mathrm{He}$ liquids, neutron matter at low density and trapped Fermi gases. In Sec. VII we describe the behavior of Fermi systems which approach FCQPT from the disordered phase. In the vicinity of FCQPT, this behavior can be viewed as a highly correlated one, because the effective mass is large and strongly depends on the density. Finally, in Sec. VIII, we summarize our main results.

\section{NORMAL STATE OF FERMI LIQUIDS WITH FC}

Let us start by explaining the important points of the FC theory, which is a special solution of the Fermi-liquid theory equations [3] for the quasiparticle occupation numbers $n(p, T)$,

$$
\frac{\delta(F-\mu N)}{\delta n(p, T)}=\varepsilon(p, T)-\mu(T)-T \ln \frac{1-n(p, T)}{n(p, T)}=0,
$$

which depends on the momentum $p$ and temperature $T$. Here $F=E-T S$ is the free energy, $S$ is the entropy, and $\mu$ is the chemical potential, while

$$
\varepsilon(p, T)=\frac{\delta E[n(p, T)]}{\delta n(p, T)}
$$

is the quasiparticle energy. This energy is a functional of $n(p, T)$ just like the total energy $E[n(p, T)]$, entropy $S[n(p, T)]$, and other thermodynamic functions. The entropy $S[n(p, T)]$ is given by the familiar expression

$$
S[n(p, T)]=-\sum_{p}[n(p, T) \ln n(p, T)+(1-n(p, T)) \ln (1-n(p, T))]
$$


which stems from purely combinatorial considerations. Eq. (1) is usually presented as the Fermi-Dirac distribution

$$
n(p, T)=\left\{1+\exp \left[\frac{(\varepsilon(p, T)-\mu)}{T}\right]\right\}^{-1} .
$$

At $T \rightarrow 0$, one gets from Eqs. (1) and (3) the standard solution $n_{F}(p, T \rightarrow 0) \rightarrow \theta\left(p_{F}-p\right)$, with $\varepsilon\left(p \simeq p_{F}\right)-\mu=p_{F}\left(p-p_{F}\right) / M_{L}^{*}$, where $p_{F}$ is the Fermi momentum and $M_{L}^{*}$ is the Landau effective mass $[3]$

$$
\frac{1}{M_{L}^{*}}=\left.\frac{1}{p} \frac{d \varepsilon(p, T=0)}{d p}\right|_{p=p_{F}} .
$$

It is implied that $M_{L}^{*}$ is positive and finite at the Fermi momentum $p_{F}$. As a result, the $T$-dependent corrections to $M_{L}^{*}$, to the quasiparticle energy $\varepsilon(p)$, and to other quantities, start with $T^{2}$-terms. But this solution is not the only one possible. There exist also "anomalous" solutions of Eq. (1) associated with the so-called fermion condensation [4,7]. Being continuous and satisfying the inequality $0<n(p)<1$ within some region in $p$, such solutions $n(p)$ admit a finite value for the logarithm in Eq. (1) at $T \rightarrow 0$ yielding

$$
\varepsilon(p)=\frac{\delta E[n(p)]}{\delta n(p)}=\mu ; \quad p_{i} \leq p \leq p_{f} .
$$

At $T=0$, Eq. (5) determines FCQPT, possessing solutions at some density $x=x_{F C}$ as soon as the effective inter-fermion interaction becomes sufficiently strong [7,8]. For instance, in the ordinary electron liquid, the effective inter-electron interaction is proportional to the dimensionless average interparticle distance $r_{s}=r_{0} / a_{B}$, with $r_{0} \sim 1 / p_{F}$ being the average distance and $a_{B}$ is the Bohr radius. When fermion condensation can take place at $r_{s}>1$, it is considered to be in the low density electron liquid [8].

Equation (5) leads to the minimal value of $E$, as a functional of $n(p)$, when a strong rearrangement of the single particle spectra can take place in the system under consideration. We see from Eq. (5) that the occupation numbers $n(p)$ become variational parameters: the FC solution appears if the energy $E$ can be lowered by alteration of the occupation numbers $n(p)$. Thus, within the region $p_{i}<p<p_{f}$, the solution $n(p)=n_{F}(p)+\delta n(p)$ deviates from the Fermi step function $n_{F}(p)$ in such a way that the energy $\varepsilon(p)$ stays constant while outside this region $n(p)$ coincides with $n_{F}(p)$. It is essential to note that the general consideration presented above has been verified by inspecting some simple models. As a result, it was shown that the onset of the FC does lead to lowering of the free energy $[7,9]$.

It follows from the above consideration that the superconductivity order parameter $\kappa(\mathbf{p})=$ $\sqrt{n(\mathbf{p})(1-n(\mathbf{p}))}$ has a nonzero value over the region occupied by FC. The superconducting gap $\Delta(\mathbf{p})$ being linear in the coupling constant of the particle-particle interaction $V\left(\mathbf{p}_{1}, \mathbf{p}_{2}\right)$ increases the value of $T_{c}$ because one has $2 T_{c} \simeq \Delta_{1}$ [9] within the standard Bardeen-Cooper-Schrieffer (BCS) theory [10]. As shown in Sec. III, if the superconducting gap is non-zero, $\Delta_{1} \neq 0$, the FC quasiparticle effective mass becomes finite. Consequently, the density of states at the Fermi level becomes finite and the quasiparticles involved are delocalized. On the other hand, even at $T=0, \Delta_{1}$ can vanish, provided the interparticle interaction $V\left(\mathbf{p}_{1}, \mathbf{p}_{2}\right)$ is either repulsive or absent. Then, as seen from Eq. (5), the Landau quasiparticle system becomes separated into two subsystems. The first contains the 
Landau quasiparticles, while the second, related to FC, is localized at the Fermi surface and is formed by dispersionless quasiparticles. As a result, beyond the point of the FC phase transition the standard Kohn-Sham scheme for the single-particle equations is no longer valid [11]. Such a behavior of systems with FC is clearly different from what one expects from the well known local density approach. Therefore, this in generally a very powerful method is hardly applicable for the description of systems with FC. It is also seen from Eq. (5) that a system with FC has a well-defined Fermi surface.

Let us assume that with the decrease of the density or growth of the interaction strength FC has just taken place. It means that $p_{i} \rightarrow p_{f} \rightarrow p_{F}$, and the deviation $\delta n(p)$ is small. Expanding the functional $E[n(p)]$ in Taylor's series with respect to $\delta n(p)$ and retaining the leading terms, one obtains from Eq. (5) the following relation

$$
\mu=\varepsilon(\mathbf{p})=\varepsilon_{0}(\mathbf{p})+\int F_{L}\left(\mathbf{p}, \mathbf{p}_{1}\right) \delta n\left(\mathbf{p}_{\mathbf{1}}\right) \frac{d \mathbf{p}_{1}}{(2 \pi)^{2}} ; \quad p_{i} \leq p \leq p_{f}
$$

where $F_{L}\left(\mathbf{p}, \mathbf{p}_{1}\right)=\delta^{2} E / \delta n(\mathbf{p}) \delta n\left(\mathbf{p}_{1}\right)$ is the Landau effective interaction. Both quantities, the interaction and the single-particle energy $\varepsilon_{0}(p)$ are calculated at $n(p)=n_{F}(p)$. Equation (6) acquires nontrivial solutions at some density $x=x_{F C}$ and FCQPT takes place if the Landau amplitudes depending on the density are positive and sufficiently large, so that the potential energy is bigger than the kinetic energy. Then the transformation of the Fermi step function $n(p)=\theta\left(p_{F}-p\right)$ into the smooth function defined by Eq. (5) becomes possible [4,7]. It is seen from Eq. (5) that the FC quasiparticles form a collective state, since their energies are defined by the macroscopical number of quasiparticles within the momentum region $p_{i}-p_{f}$. The shape of the excitation spectra related to FC is not affected by the Landau interaction, which, generally speaking, depends on the system's properties, including the collective states, impurities, etc. The only thing determined by the interaction is the width of the FC region $p_{i}-p_{f}$ provided the interaction is sufficiently strong to produce the FC phase transition at all. Thus, we can conclude that the spectra related to FC are of a universal form, being dependent, as we will see below, mainly on temperature $T$ if $T>T_{c}$ or on the superconducting gap at $T<T_{c}$.

According to Eq. (1), the single-particle excitations $\varepsilon(p, T)$ within the interval $p_{i}-p_{f}$ at $T_{c} \leq$ $T \ll T_{f}$ are linear in $T$, which can be simplified at the Fermi level [12]. One obtains by expanding $\ln (\ldots)$ in terms of $n(p)$

$$
\varepsilon(p, T)-\mu(T)=\left.T \ln \frac{1-n(p)}{n(p)} \simeq T \frac{1-2 n(p)}{n(p)}\right|_{p \simeq p_{F}} .
$$

Here $T_{f}$ is the temperature, above which FC effects become insignificant [9],

$$
\frac{T_{f}}{\varepsilon_{F}} \sim \frac{p_{f}^{2}-p_{i}^{2}}{2 M \varepsilon_{F}} \sim \frac{\Omega_{F C}}{\Omega_{F}} .
$$

In this formula $\Omega_{F C}$ is the FC volume, $\varepsilon_{F}$ is the Fermi energy, and $\Omega_{F}$ is the volume of the Fermi sphere. We note that at $T_{c} \leq T \ll T_{f}$ the occupation numbers $n(p)$ are approximately independent of $T$, being given by Eq. (5). According to Eq. (1), the dispersionless plateau $\varepsilon(p)=\mu$ is slightly turned counter-clockwise about $\mu$. As a result, the plateau is just a little tilted and rounded off at the end points. According to Eq. (7) the effective mass $M_{F C}^{*}$ related to $\mathrm{FC}$ is given by,

$$
M_{F C}^{*} \simeq p_{F} \frac{p_{f}-p_{i}}{4 T}
$$


To obtain Eq. (9) an approximation for the derivative $d n(p) / d p \simeq-1 /\left(p_{f}-p_{i}\right)$ was used.

Having in mind that $\left(p_{f}-p_{i}\right) \ll p_{F}$ and using Eqs. (8) and (9), the following estimates for the effective mass $M_{F C}^{*}$ are obtained:

$$
\frac{M_{F C}^{*}}{M} \sim \frac{N(0)}{N_{0}(0)} \sim \frac{T_{f}}{T} .
$$

Eqs. (9) and (10) show the temperature dependence of $M_{F C}^{*}$. In Eq. (10) $M$ denotes the bare electron mass, $N_{0}(0)$ is the density of states of noninteracting electron gas, and $N(0)$ is the density of states at the Fermi level. Multiplying both sides of Eq. (9) by $\left(p_{f}-p_{i}\right)$ we obtain the energy scale $E_{0}$ separating the slow dispersing low energy part related to the effective mass $M_{F C}^{*}$ from the faster dispersing relatively high energy part defined by the effective mass $M_{L}^{*}[6,13]$,

$$
E_{0} \simeq 4 T \text {. }
$$

It is seen from Eq. (11) that the scale $E_{0}$ does not depend on the condensate volume. The single particle excitations are defined according to Eq. (9) by the temperature and by $\left(p_{f}-p_{i}\right)$, given by Eq. (5). Thus, we conclude that the one-electron spectrum is negligibly disturbed by thermal excitations, impurities, etc, which are the features of the "quantum protectorate" [14,15].

It is pertinent to note that outside the $\mathrm{FC}$ region the single particle spectrum is not affected by the temperature, being defined by $M_{L}^{*}$. Thus we come to the conclusion that a system with $\mathrm{FC}$ is characterized by two effective masses: $M_{F C}^{*}$ which is related to the single particle spectrum at lower energy scale and $M_{L}^{*}$ describing the spectrum at higher energy scale. The existence of two effective masses is manifested by a break (or kink) in the quasiparticle dispersion, which can be approximated by two straight lines intersecting at the energy $E_{0}$. This break takes place at temperatures $T_{c} \leq T \ll T_{f}$, in accord with the experimental data [16], and, as we will see, at $T \leq T_{c}$ which is also in accord with the experimental facts $[16,17]$. The quasiparticle formalism is applicable to this problem since the width $\gamma$ of single particle excitations is not large compared to their energy, being proportional to the temperature, $\gamma \sim T$ at $T>T_{c}$ [9]. The lineshape can be approximated by a simple Lorentzian [13], consistent with experimental data obtained from scans at a constant binding energy [18] (see Sec. IV).

It is seen from Eq. (5) that at the point of FC phase transition $p_{f} \rightarrow p_{i} \rightarrow p_{F}, M_{F C}^{*}$ and the density of states, as it follows from Eqs. (5) and (10), tend to infinity. One can conclude that at $T=0$ and as soon as $x \rightarrow x_{F C}$, FCQPT takes place being connected to the absolute growth of $M_{L}^{*}$.

It is essential to have in mind, that the onset of the charge density wave instability in a manyelectron system, such as an electron liquid, which takes place as soon as the effective inter-electron constant reaches its critical value $r_{s}=r_{c d w}$, is preceded by the unlimited growth of the effective mass, see Sec. VI. Therefore the FC occurs before the onset of the charge density wave. Hence, at $T=0$, when $r_{s}$ reaches its critical value $r_{F C}$ corresponding to $x_{F C}, r_{F C}<r_{c d w}$, FCQPT inevitably takes place [8]. It is pertinent to note that this growth of the effective mass with decreasing electron density was observed experimentally in a metallic $2 \mathrm{D}$ electron system in silicon at $r_{s} \simeq 7.5$ [19]. Therefore we can take $r_{F C} \sim 7.5$. On the other hand, there exist charge density waves or strong fluctuations of charge ordering in underdoped high- $T_{c}$ superconductors [20]. Thus the formation of FC in high- $T_{c}$ compounds can be thought as a general property of an electron liquid of low density which is embedded in these solids rather than an uncommon and anomalous solution of Eq. (1) [8]. Beyond the point of FCQPT, the condensate volume is proportional to $\left(r_{s}-r_{F C}\right)$ as well as $T_{f} / \varepsilon_{F} \sim\left(r_{s}-r_{F C}\right) / r_{F C}$ at least when $\left(r_{s}-r_{F C}\right) / r_{F C} \ll 1$, and we obtain 


$$
\frac{r_{s}-r_{F C}}{r_{F C}} \sim \frac{p_{f}-p_{i}}{p_{F}} \sim \frac{x_{F C}-x}{x_{F C}}
$$

FC serves as a stimulator that creates new phase transitions, which lift the degeneration of the spectrum. For example FC can generate spin density waves or antiferromagnetic phase transition, thus leading to a whole variety of new properties of the system under consideration. Then, the onset of the charge density wave is preceded by FCQPT, and both of these phases can coexist at the sufficiently low density when $r_{s} \geq r_{c d w}$.

We have demonstrated above that superconductivity is strongly aided by FC because both of the phases are characterized by the same order parameter. As a result, the superconductivity removing the spectrum degeneration, "wins" the competition with the other phase transitions up to the critical temperature $T_{c}$. We now turn to the consideration of the superconducting state and quasiparticle dispersions at $T \leq T_{c}$.

\section{THE SUPERCONDUCTING STATE}

At $T=0$, the ground state energy $E_{g s}[\kappa(\mathbf{p}), n(\mathbf{p})]$ of a $2 \mathrm{D}$ electron liquid is a functional of the order parameter of the superconducting state $\kappa(\mathbf{p})$ and of the quasiparticle occupation numbers $n(\mathbf{p})$. This energy is determined by the known equation of the weak-coupling theory of superconductivity, see e.g. [21]

$$
E_{g s}=E[n(\mathbf{p})]+\int \lambda_{0} V\left(\mathbf{p}_{1}, \mathbf{p}_{2}\right) \kappa\left(\mathbf{p}_{1}\right) \kappa^{*}\left(\mathbf{p}_{2}\right) \frac{d \mathbf{p}_{1} d \mathbf{p}_{2}}{(2 \pi)^{4}}
$$

Here $E[n(\mathbf{p})]$ is the ground-state energy of a normal Fermi liquid, $n(\mathbf{p})=v^{2}(\mathbf{p})$ and $\kappa(\mathbf{p})=$ $v(\mathbf{p}) \sqrt{1-v^{2}(\mathbf{p})}$. It is assumed that the pairing interaction $\lambda_{0} V\left(\mathbf{p}_{1}, \mathbf{p}_{2}\right)$ is weak. Minimizing $E_{g s}$ with respect to $\kappa(\mathbf{p})$ we obtain the equation connecting the single-particle energy $\varepsilon(\mathbf{p})$ to $\Delta(\mathbf{p})$,

$$
\varepsilon(\mathbf{p})-\mu=\Delta(\mathbf{p}) \frac{1-2 v^{2}(\mathbf{p})}{2 \kappa(\mathbf{p})},
$$

where the single-particle energy $\varepsilon(\mathbf{p})$ is determined by the Landau equation (2). The equation for the superconducting gap $\Delta(\mathbf{p})$ takes the form

$$
\begin{aligned}
\Delta(\mathbf{p}) & =-\int \lambda_{0} V\left(\mathbf{p}, \mathbf{p}_{1}\right) \kappa\left(\mathbf{p}_{1}\right) \frac{d \mathbf{p}_{1}}{4 \pi^{2}} \\
& =-\frac{1}{2} \int \lambda_{0} V\left(\mathbf{p}, \mathbf{p}_{1}\right) \frac{\Delta\left(\mathbf{p}_{1}\right)}{\sqrt{\left(\varepsilon\left(\mathbf{p}_{1}\right)-\mu\right)^{2}+\Delta^{2}\left(\mathbf{p}_{1}\right)}} \frac{d \mathbf{p}_{1}}{4 \pi^{2}} .
\end{aligned}
$$

If $\lambda_{0} \rightarrow 0$, then the maximum value $\Delta_{1} \rightarrow 0$ and Eq. (14) reduces to Eq. (5) [4]

$$
\varepsilon(\mathbf{p})-\mu=0, \quad \text { if } \quad 0<n(\mathbf{p})<1 ; p_{i} \leq p \leq p_{f} .
$$

Now we can study the relationships between the state defined by Eq. (16), or by Eq. (5), and the superconductivity. At $T=0$, Eq. (16) defines a particular state of a Fermi-liquid with FC, for which the modulus of the order parameter $|\kappa(\mathbf{p})|$ has finite values in the $L_{F C}$ range of momenta $p_{i} \leq p \leq p_{f}$, and $\Delta_{1} \rightarrow 0$ in the $L_{F C}$. Such a state can be considered as superconducting, with an infinitely small 
value of $\Delta_{1}$, so that the entropy of this state is equal to zero. It is obvious that this state being driven by the quantum phase transition disappears at $T>0$ [6]. Any quantum phase transition, which takes place at temperature $T=0$, is determined by a control parameter other than temperature, for instance, by pressure, by magnetic field, or by the density of mobile charge carriers $x \sim 1 / r_{s}^{2}$. The quantum phase transition occurs at the quantum critical point. In a common case, this point is the end of a line of continuous transitions at $T=0$.

As any phase transition, the quantum phase transition is related to the order parameter, which induces a broken symmetry. In our case as we show in Sec. II, the control parameter is the density of a system, which determines the strength of the Landau effective interaction, and the order parameter is $\kappa(\mathbf{p})$. As we point out in Sec. $\mathrm{V}$, the existence of such a state can be revealed experimentally. Since the order parameter $\kappa(\mathbf{p})$ is suppressed by a magnetic field $B$, when $B^{2} \sim \Delta_{1}^{2}$, a weak magnetic field $B$ will destroy the state with FC converting the strongly correlated Fermi liquid into the normal Landau Fermi liquid. In this case the magnetic field play a role of the control parameter.

When $p_{i} \rightarrow p_{F} \rightarrow p_{f}$, Eq. (16) determines the critical point $r_{F C}$ at which FCQPT takes place. It follows from Eq. (16) that the system becomes divided into two quasiparticle subsystems: the first subsystem in the $L_{F C}$ range is characterized by the quasiparticles with the effective mass $M_{F C}^{*} \propto 1 / \Delta_{1}$, while the second one is occupied by quasiparticles with finite mass $M_{L}^{*}$ and momenta $p<p_{i}$. The density of states near the Fermi level tends to infinity, $N(0) \sim M_{F C}^{*} \sim 1 / \Delta_{1}$. The quasiparticles with $M_{F C}^{*}$ occupy the same energy level and form pairs with binding energy of the order of $\Delta_{1}$ and with average momentum $p_{0}, p_{0} / p_{F} \sim\left(p_{f}-p_{i}\right) / p_{F} \ll 1$. Therefore, this state strongly resembles the Bose-Einstein condensation when quasiparticles occupy the same energy level. But these have to be spread over the range $L_{F C}$ in momentum space due to the exclusion principle. In contrast to the Bose-Einstein condensation, the fermion condensation temperature is $T_{c}=0$. And in contrast to the ordinary superconductivity, the fermion condensation is driven by the Landau repulsive interaction rather than by relatively weak attractive quasiparticle-quasiparticle interaction $\lambda_{0} V\left(\mathbf{p}_{1}, \mathbf{p}_{2}\right)$.

If $\lambda_{0} \neq 0, \Delta_{1}$ becomes finite, leading to a finite value of the effective mass $M_{F C}^{*}$ in $L_{F C}$, which can be obtained from Eq. (14) [6,13]

$$
M_{F C}^{*} \simeq p_{F} \frac{p_{f}-p_{i}}{2 \Delta_{1}}
$$

As to the energy scale it is determined by the parameter $E_{0}$ :

$$
E_{0}=\varepsilon\left(\mathbf{p}_{f}\right)-\varepsilon\left(\mathbf{p}_{i}\right) \simeq 2 \frac{\left(p_{f}-p_{F}\right) p_{F}}{M_{F C}^{*}} \simeq 2 \Delta_{1} .
$$

It is natural to assume that we have returned back to the Landau Fermi-liquid theory eliminating high energy degrees of freedom and introducing the quasiparticles. The only difference between the Landau Fermi-liquid and Fermi-liquid after FCQPT is that we have to expand the number of relevant low energy degrees of freedom by introducing new type of quasiparticles with the effective mass $M_{F C}^{*}$ given by Eq. (17) and the energy scale $E_{0}$ given by Eq. (18). Properties of these new quasiparticles are closely related to the properties of the superconducting state as it follows from Eqs. (14), (17) and (18). We may say that the quasiparticle system in the range $L_{F C}$ becomes very "soft" and is to be considered as a strongly correlated liquid. On the other hand, the system's properties and dynamics are dominated by a strong collective effect having its origin in FCQPT and determined by the macroscopic number of quasiparticles in the range $L_{F C}$. Such a system cannot be disturbed by the scattering of individual quasiparticles and has features of a "quantum protectorate" $[6,14,15]$. 
We assume that the range $L_{F C}$ is small, $\left(p_{f}-p_{F}\right) / p_{F} \ll 1$, and $2 \Delta_{1} \ll T_{f}$ so that the order parameter $\kappa(\mathbf{p})$ is governed mainly by FC [6,26]. To solve Eq. (15) analytically, we take the BardeenCooper-Schrieffer (BCS) approximation for the interaction [10]: $\lambda_{0} V\left(\mathbf{p}, \mathbf{p}_{1}\right)=-\lambda_{0}$ if $|\varepsilon(\mathbf{p})-\mu| \leq \omega_{D}$, i.e. the interaction is zero outside this region, with $\omega_{D}$ being the characteristic phonon energy. As a result, the gap becomes dependent only on the temperature, $\Delta(\mathbf{p})=\Delta_{1}(T)$, being independent of the momentum, and Eq. (15) takes the form

$$
1=N_{F C} \lambda_{0} \int_{0}^{E_{0} / 2} \frac{d \xi}{\sqrt{\xi^{2}+\Delta_{1}^{2}(0)}}+N_{L} \lambda_{0} \int_{E_{0} / 2}^{\omega_{D}} \frac{d \xi}{\sqrt{\xi^{2}+\Delta_{1}^{2}(0)}} .
$$

Here we set $\xi=\varepsilon(\mathbf{p})-\mu$ and introduce the density of states $N_{F C}$ in the $L_{F C}$, or $E_{0}$, range. It follows from Eq. (17), $N_{F C}=\left(p_{f}-p_{F}\right) p_{F} / 2 \pi \Delta_{1}(0)$. The density of states $N_{L}$ in the range $\left(\omega_{D}-E_{0} / 2\right)$ has the standard form $N_{L}=M_{L}^{*} / 2 \pi$. If the energy scale $E_{0} \rightarrow 0$, Eq. (19) reduces to the BCS equation. On the other hand, assuming that $E_{0} \leq 2 \omega_{D}$ and omitting the second integral on the right hand side of Eq. (19), we obtain

$$
\Delta_{1}(0)=\frac{\lambda_{0} p_{F}\left(p_{f}-p_{F}\right)}{2 \pi} \ln (1+\sqrt{2})=2 \beta \varepsilon_{F} \frac{p_{f}-p_{F}}{p_{F}} \ln (1+\sqrt{2})
$$

where the Fermi energy $\varepsilon_{F}=p_{F}^{2} / 2 M_{L}^{*}$, and the dimensionless coupling constant $\beta$ is given by the relation $\beta=\lambda_{0} M_{L}^{*} / 2 \pi$. Taking the usual values of $\beta$ as $\beta \simeq 0.3$, and assuming $\left(p_{f}-p_{F}\right) / p_{F} \simeq 0.2$, we get from Eq. (20) a large value of $\Delta_{1}(0) \sim 0.1 \varepsilon_{F}$, while for normal metals one has $\Delta_{1}(0) \sim 10^{-3} \varepsilon_{F}$. Taking into account the omitted integral, we obtain

$$
\Delta_{1}(0) \simeq 2 \beta \varepsilon_{F} \frac{p_{f}-p_{F}}{p_{F}} \ln (1+\sqrt{2})\left(1+\beta \ln \frac{2 \omega_{D}}{E_{0}}\right)
$$

It is seen from Eq. (21) that the correction due to the second integral is small, provided $E_{0} \simeq 2 \omega_{D}$. Below we show that $2 T_{c} \simeq \Delta_{1}(0)$, which leads to the conclusion that there is no isotope effect since $\Delta_{1}$ is independent of $\omega_{D}$. But this effect is restored as $E_{0} \rightarrow 0$. Assuming $E_{0} \sim \omega_{D}$ and $E_{0}>\omega_{D}$, we see that Eq. (21) has no standard solutions $\Delta(p)=\Delta_{1}(T=0)$ because $\omega_{D}<\varepsilon\left(p \simeq p_{f}\right)-\mu$ and the interaction vanishes at these momenta. The only way to obtain solutions is to restore the condition $E_{0}<\omega_{D}$. For instance, we can define such a momentum $p_{D}<p_{f}$ that

$$
\Delta_{1}(0)=2 \beta \varepsilon_{F} \frac{p_{D}-p_{F}}{p_{F}} \ln (1+\sqrt{2})=\omega_{D}
$$

while the other part in the $L_{F C}$ range can be occupied by a gap $\Delta_{2}$ of the different sign, $\Delta_{1} / \Delta_{2}<0$. It follows from Eq. (22) that the isotope effect is preserved, while both gaps can have $s$-wave symmetry.

At $T \simeq T_{c}$ Eqs. (17) and (18) are replaced by the equation, which is valid also at $T_{c} \leq T \ll T_{f}$ in accord with Eq. (9) [6]:

$$
M_{F C}^{*} \simeq p_{F} \frac{p_{f}-p_{i}}{4 T_{c}}, \quad E_{0} \simeq 4 T_{c} ; \text { if } T_{c} \leq T \text { and } M_{F C}^{*} \simeq p_{F} \frac{p_{f}-p_{i}}{4 T}, E_{0} \simeq 4 T
$$

Equation (19) is replaced by its conventional finite temperature generalization 


$$
\begin{aligned}
1 & =N_{F C} \lambda_{0} \int_{0}^{E_{0} / 2} \frac{d \xi}{\sqrt{\xi^{2}+\Delta_{1}^{2}(T)}} \tanh \frac{\sqrt{\xi^{2}+\Delta_{1}^{2}(T)}}{2 T}+ \\
& +N_{L} \lambda_{0} \int_{E_{0} / 2}^{\omega_{D}} \frac{d \xi}{\sqrt{\xi^{2}+\Delta_{1}^{2}(T)}} \tanh \frac{\sqrt{\xi^{2}+\Delta_{1}^{2}(T)}}{2 T} .
\end{aligned}
$$

Putting $\Delta_{1}\left(T \rightarrow T_{c}\right) \rightarrow 0$, we obtain from Eq. (24)

$$
2 T_{c} \simeq \Delta_{1}(0),
$$

with $\Delta_{1}(T=0)$ being given by Eq. (20). Comparing Eqs. (17), (23) and (25), we see that $M_{F C}^{*}$ and $E_{0}$ are almost temperature independent at $T \leq T_{c}$.

Now let us comment about some special features of the superconducting state with FC. One can define $T_{c}$ as the temperature when $\Delta_{1}\left(T_{c}\right) \equiv 0$. At $T \geq T_{c}$ Eq. (24) has only the trivial solution $\Delta_{1} \equiv 0$. On the other hand, $T_{c}$ can be defined as a temperature, at which the superconductivity disappears. Thus, we have two different definitions, which can lead to two different temperatures $T_{c}$ and $T^{*}$ in case of the $d$-wave symmetry of the gap. It was shown $[13,22]$ that in the case of the $\mathbf{d}$-wave superconductivity in the presence of FC there is a nontrivial solution of Eq. (24) at $T_{c} \leq T \leq T^{*}$ corresponding to the pseudogap state. It happens when the gap occupies only such a part of the Fermi surface, which shrinks as the temperature increases. Here $T^{*}$ defines the temperature at which $\Delta_{1}\left(T^{*}\right) \equiv 0$ and the pseudogap state vanishes. The superconductivity is destroyed at $T_{c}$, and the ratio $2 \Delta_{1} / T_{c}$ can vary in a wide range and strongly depends upon the material's properties as it follows from considerations given in $[13,22,23]$. Therefore, if a pseudogap exists above $T_{c}$, then $T_{c}$ is to be replaced by $T^{*}$ and Eq. (25) takes the form

$$
2 T^{*} \simeq \Delta_{1}(0) .
$$

The ratio $2 \Delta_{1} / T_{c}$ can reach very high values. For instance, in the case of $\mathrm{Bi}_{2} \mathrm{Sr}_{2} \mathrm{CaCu}_{2} \mathrm{Q}_{6+\delta}$ where the superconductivity and the pseudogap are considered to be of the common origin, $2 \Delta_{1} / T_{c}$ is about 28 , while the ratio $2 \Delta_{1} / T^{*} \simeq 4$, which is in agreement with the experimental data for various cuprates [24]. Note that Eq. (20) gives also good description of the maximum gap $\Delta_{1}$ in the case of the d-wave superconductivity, because the different regions with the maximum absolute value of $\Delta_{1}$ and the maximal density of states can be considered as disconnected [25]. Therefore the gap in this region is formed by attractive phonon interaction, which is approximately independent of the momenta.

Consider now two possible types of the superconducting gap $\Delta(\mathbf{p})$ given by Eq. (15) and defined by the interaction $\lambda_{0} V\left(\mathbf{p}, \mathbf{p}_{1}\right)$. If this interaction is dominated by a phonon-mediated attraction, the even solution of Eq. (15) with the $s$-wave, or the $s+d$ mixed waves will have the lowest energy. Provided the pairing interaction $\lambda_{0} V\left(\mathbf{p}_{1}, \mathbf{p}_{2}\right)$ is the combination of both the attractive interaction and sufficiently strong repulsive interaction, the $d$-wave odd superconductivity can take place (see e.g. [25]). But both the $s$-wave even symmetry and the $d$-wave odd one lead to approximately the same value of the gap $\Delta_{1}$ in Eq. (21) [26]. Therefore the non-universal pairing symmetries in high- $T_{c}$ superconductivity is likely the result of the pairing interaction and the $d$-wave pairing symmetry is not essential. This point of view is supported by the data [27-31]. If only the $d$-wave pairing would exist, the transition from superconducting gap to pseudogap could take place, so that the superconductivity would be destroyed at $T_{c}$, with the superconducting gap being smoothly transformed into the pseudogap, which closes at some temperature $T^{*}>T_{c}[22,23]$. In the case of the $s$-wave pairing we can expect the absence of 
the pseudogap phenomenon in accordance with the experimental observation (see [31] and references therein).

We now turn to a consideration of the maximum value of the superconducting gap $\Delta_{1}$ as a function of the density $x$ of the mobile charge carriers. Rewritting in terms of $x \sim r_{s}^{2}$ and $x_{F C} \sim r_{F C}^{2}$ which are related to the variables $p_{i}$ and $p_{f}$ by Eq. (12), Eq. (21) becomes

$$
\Delta_{1} \propto \beta\left(x_{F C}-x\right) x .
$$

Here we take into account that the Fermi level $\varepsilon_{F} \propto p_{F}^{2}$, the density $x \propto p_{F}^{2}$, and thus, $\varepsilon_{F} \propto x$. We can reliably assume that $T_{c} \propto \Delta_{1}$ because the empirically obtained simple bell-shaped curve of $T_{c}(x)$ in the high temperature superconductors [32] should have only a smooth dependence. Then, $T_{c}(x)$ in accordance with the data has the form [33]

$$
T_{c}(x) \propto \beta\left(x_{F C}-x\right) x .
$$

As an example of the implementation of the previous analysis, let us consider the main features of a room-temperature superconductor. The superconductor has to be a quasi two-dimensional structure like cuprates. From Eq. (21) it follows, that $\Delta_{1} \sim \beta \varepsilon_{F} \propto \beta / r_{s}^{2}$. Noting that FCQPT in 3D systems takes place at $r_{s} \sim 20$ and in 2D systems at $r_{s} \sim 8$ [8], we can expect that $\Delta_{1}$ of 3D systems comprises $10 \%$ of the corresponding maximum value of $2 \mathrm{D}$ superconducting gap, reaching a value as high as $60 \mathrm{meV}$ for underdoped crystals with $T_{c}=70$ [34]. On the other hand, it is seen from Eq. (21), that $\Delta_{1}$ can be even large, $\Delta_{1} \sim 75 \mathrm{meV}$, and one can expect $T_{c} \sim 300 \mathrm{~K}$ in the case of the $s$ wave pairing as it follows from the simple relation $2 T_{c} \simeq \Delta_{1}$. In fact, we can safely take $\varepsilon_{F} \sim 300 \mathrm{meV}$, $\beta \sim 0.5$ and $\left(p_{f}-p_{i}\right) / p_{F} \sim 0.5$. Thus, a possible room-temperature superconductor has to be the $s$-wave superconductor in order to get rid of the pseudogap phenomena, which tremendously reduces the transition temperature. The density $x$ of the mobile charge carriers must satisfy the condition $x \leq x_{F C}$ and be flexible to reach the optimal doping level $x_{\text {opt }} \simeq x_{F C} / 2$.

Now we turn to the calculations of the gap and the specific heat at the temperatures $T \rightarrow T_{c}$. It is worth noting that this consideration is valid provided $T^{*}=T_{c}$, otherwise the considered below discontinuity is smoothed out over the temperature range $T^{*} \div T_{c}$. For the sake of simplicity, we calculate the main contribution to the gap and the specific heat coming from the FC. The function $\Delta_{1}\left(T \rightarrow T_{c}\right)$ is found from Eq. (24) by expanding the right hand side of the first integral in powers of $\Delta_{1}$ and omitting the contribution from the second integral on the right hand side of Eq. (24). This procedure leads to the following equation [26]

$$
\Delta_{1}(T) \simeq 3.4 T_{c} \sqrt{1-\frac{T}{T_{c}}} .
$$

Thus, the gap in the spectrum of the single-particle excitations has the usual behavior. To calculate the specific heat, the conventional expression for the entropy $S$ [10] can be used

$$
S=-2 \int[f(\mathbf{p}) \ln f(\mathbf{p})+(1-f(\mathbf{p})) \ln (1-f(\mathbf{p}))] \frac{d \mathbf{p}}{(2 \pi)^{2}},
$$

where

$$
f(\mathbf{p})=\frac{1}{1+\exp [E(\mathbf{p}) / T]} ; \quad E(\mathbf{p})=\sqrt{(\varepsilon(\mathbf{p})-\mu)^{2}+\Delta_{1}^{2}(T)} .
$$


The specific heat $C$ is determined by the equation

$$
\begin{aligned}
C & =T \frac{d S}{d T} \simeq 4 \frac{N_{F C}}{T^{2}} \int_{0}^{E_{0}} f(E)(1-f(E))\left[E^{2}+T \Delta_{1}(T) \frac{d \Delta_{1}(T)}{d T}\right] d \xi+ \\
& +4 \frac{N_{L}}{T^{2}} \int_{E_{0}}^{\omega_{D}} f(E)(1-f(E))\left[E^{2}+T \Delta_{1}(T) \frac{d \Delta_{1}(T)}{d T}\right] d \xi .
\end{aligned}
$$

In deriving Eq. (32) we again used the variable $\xi$ and the densities of states $N_{F C}$ and $N_{L}$, just as before in connection with Eq. (21), and employed the notation $E=\sqrt{\xi^{2}+\Delta_{1}^{2}(T)}$. Eq. (32) predicts the conventional discontinuity $\delta C$ in the specific heat $C$ at $T_{c}$ because of the last term in the square brackets of Eq. (32). Using Eq. (29) to calculate this term and omitting the second integral on the right hand side of Eq. (32), we obtain

$$
\delta C \simeq \frac{3}{2 \pi}\left(p_{f}-p_{i}\right) p_{F} .
$$

This is in contrast to the conventional result where the discontinuity is a linear function of $T_{c}$. $\delta C$ is independent of the critical temperature $T_{c}$ because as seen from Eq. (23) the density of states varies inversely with $T_{c}$. Note, that in deriving Eq. (33) we took into account the main contribution coming from the FC. This term vanishes as soon as $E_{0} \rightarrow 0$ and the second integral of Eq. (32) gives the conventional result.

\section{THE LINESHAPE OF THE SINGLE-PARTICLE SPECTRA}

The lineshape $L(q, \omega)$ of the single-particle spectrum is a function of two variables. Measurements carried out at a fixed binding energy $\omega=\omega_{0}$, with $\omega_{0}$ being the energy of a single-particle excitation, determine the lineshape $L\left(q, \omega=\omega_{0}\right)$ as a function of the momentum $q$. We have shown above that $M_{F C}^{*}$ is finite and constant at $T \leq T_{c}$. Therefore, at excitation energies $\omega \leq E_{0}$, the system behaves like an ordinary superconducting Fermi liquid with the effective mass given by Eq. (17) [6,13]. At $T_{c} \leq T$ the low energy effective mass $M_{F C}^{*}$ is finite and is given by Eq. (9). Once again, at the energies $\omega<E_{0}$, the system behaves as a Fermi liquid, the single-particle spectrum is well defined while the width of single-particle excitations is of the order of $T$ [6,9]. This behavior was observed in experiments measuring the lineshape at a fixed energy $[18,35]$.

The lineshape can also be determined as a function $L\left(q=q_{0}, \omega\right)$ at a fixed $q=q_{0}$. At small $\omega$, the lineshape resembles the one considered above, and $L\left(q=q_{0}, \omega\right)$ has the characteristic maximum and width. At energies $\omega \geq E_{0}$, the quasiparticles with the mass $M_{L}^{*}$ become important, leading to the increase of $L\left(q=q_{0}, \omega\right)$. As a result, the function $L\left(q=q_{0}, \omega\right)$ possesses the known peak-dip-hump structure [36] directly defined by the existence of the two effective masses $M_{F C}^{*}$ and $M_{L}^{*}[6,13]$. We can conclude that in contrast to the Landau quasiparticles, these quasiparticles have a more complicated lineshape.

To develop deeper quantitative and analytical insight into the problem, we use the Kramers-Krönig transformation to construct the imaginary part $\operatorname{Im} \Sigma(\mathbf{p}, \varepsilon)$ of the self-energy $\Sigma(\mathbf{p}, \varepsilon)$ starting with the real one $\operatorname{Re} \Sigma(\mathbf{p}, \varepsilon)$, which defines the effective mass [37]

$$
\frac{1}{M^{*}}=\left(\frac{1}{M}+\frac{1}{p_{F}} \frac{\partial \operatorname{Re} \Sigma}{\partial p}\right) /\left(1-\frac{\partial \operatorname{Re} \Sigma}{\partial \varepsilon}\right) .
$$


Here $M$ is the bare mass, while the relevant momenta $p$ and energies $\varepsilon$ obey the following strong inequalities: $\left|p-p_{F}\right| / p_{F} \ll 1$, and $\varepsilon / \varepsilon_{F} \ll 1$. We take $\operatorname{Re} \Sigma(\mathbf{p}, \varepsilon)$ in the simplest form which accounts for the change of the effective mass at the energy scale $E_{0}$ :

$$
\operatorname{Re} \Sigma(\mathbf{p}, \varepsilon)=-\varepsilon \frac{M_{F C}^{*}}{M}+\left(\varepsilon-\frac{E_{0}}{2}\right) \frac{M_{F C}^{*}-M_{L}^{*}}{M}\left[\theta\left(\varepsilon-\frac{E_{0}}{2}\right)+\theta\left(-\varepsilon-\frac{E_{0}}{2}\right)\right] .
$$

Here $\theta(\varepsilon)$ is the step function. Note that in order to ensure a smooth transition from the single-particle spectrum characterized by $M_{F C}^{*}$ to the spectrum defined by $M_{L}^{*}$ the step function is to be substituted by some smooth function. Upon inserting Eq. (35) into Eq. (34) we can check that inside the interval $\left(-E_{0} / 2, E_{0} / 2\right)$ the effective mass $M^{*} \simeq M_{F C}^{*}$, and outside the interval $M^{*} \simeq M_{L}^{*}$. By applying the Kramers-Krönig transformation to $\operatorname{Re} \Sigma(\mathbf{p}, \varepsilon)$, we obtain the imaginary part of the self-energy [26]

$$
\operatorname{Im} \Sigma(\mathbf{p}, \varepsilon) \sim \varepsilon^{2} \frac{M_{F C}^{*}}{\varepsilon_{F} M}+\frac{M_{F C}^{*}-M_{L}^{*}}{M}\left(\varepsilon \ln \left|\frac{\varepsilon+E_{0} / 2}{\varepsilon-E_{0} / 2}\right|+\frac{E_{0}}{2} \ln \left|\frac{\varepsilon^{2}-E_{0}^{2} / 4}{E_{0}^{2} / 4}\right|\right) .
$$

We see from Eq. (36) that at $\varepsilon / E_{0} \ll 1$ the imaginary part is proportional to $\varepsilon^{2}$, at $2 \varepsilon / E_{0} \simeq 1$ $\operatorname{Im} \Sigma \sim \varepsilon$, and at $E_{0} / \varepsilon \ll 1$ the main contribution to the imaginary part is approximately constant. This is the behavior that gives rise to the known peak-dip-hump structure. It is seen from Eq. (36) that when $E_{0} \rightarrow 0$ the second term on the right hand side tends to zero and the single-particle excitations become better defined, resembling the situation in a normal Fermi-liquid, and the peakdip-hump structure eventually vanishes. On the other hand, the quasiparticle amplitude $a(\mathbf{p})$ is given by $[37]$

$$
\frac{1}{a(\mathbf{p})}=1-\frac{\partial \operatorname{Re} \Sigma(\mathbf{p}, \varepsilon)}{\partial \varepsilon}
$$

It follows from Eq. (34) that the quasiparticle amplitude $a(\mathbf{p})$ rises as the effective mass $M_{F C}^{*}$ decreases. Since, it follows from Eq. (12), $M_{F C}^{*} \sim\left(p_{f}-p_{i}\right) / p_{F} \sim\left(x_{F C}-x\right) / x_{F C}$, we have to conclude that the amplitude $a(\mathbf{p})$ rises as the level of doping increases. Thus, the single-particle excitations become better defined in highly overdoped samples. It is worth noting that such a behavior was observed experimentally in highly overdoped Bi2212 where the gap size is about $10 \mathrm{meV}$ [38]. Such a small size of the gap verifies that the region occupied by the FC is small since $E_{0} / 2 \simeq \Delta_{1}$.

\section{HEAVY-FERMION METALS}

Now we consider the behavior of a many-electron system with FC in magnetic fields, assuming that the coupling constant $\lambda_{0} \neq 0$ is infinitely small. As we have seen in Sec. III, at $T=0$ the superconducting order parameter $\kappa(\mathbf{p})$ is finite in the FC range, while the maximum value of the superconducting gap $\Delta_{1} \propto \lambda_{0}$ is infinitely small. Therefore, any small magnetic field $B \neq 0$ can be considered as a critical field and will destroy the coherence of $\kappa(\mathbf{p})$ and thus FC itself. To define the type of FC rearrangement, simple energy arguments are sufficient. On one hand, the energy gain $\Delta E_{B}$ due to the magnetic field $B$ is $\Delta E_{B} \propto B^{2}$ and tends to zero with $B \rightarrow 0$. On the other hand, occupying the finite range $L_{F C}$ in the momentum space, the formation of $\mathrm{FC}$ leads to a finite gain in the ground state energy [4]. Thus, a new ground state replacing FC should have almost the same energy as the former one. Such a state is given by the multiconnected Fermi spheres resembling an 
onion, where the smooth quasiparticle distribution function $n(\mathbf{p})$ in the $L_{F C}$ range is replaced by a multiconnected distribution $\nu(\mathbf{p})[39]$

$$
\nu(\mathbf{p})=\sum_{k=1}^{n} \theta\left(p-p_{2 k-1}\right) \theta\left(p_{2 k}-p\right) .
$$

Here the parameters $p_{i} \leq p_{1}<p_{2}<\ldots<p_{2 n} \leq p_{f}$ are adjusted to obey the normalization condition:

$$
\int_{p_{2 k}}^{p_{2 k+3}} \nu(\mathbf{p}) \frac{d \mathbf{p}}{(2 \pi)^{3}}=\int_{p_{2 k}}^{p_{2 k+3}} n(\mathbf{p}) \frac{d \mathbf{p}}{(2 \pi)^{3}} .
$$

For definiteness, let us consider the most interesting case of a 3D system, while the consideration of a 2D system also goes along the same line. We note that the idea of multiconnected Fermi spheres, with production of new, interior segments of the Fermi surface, has been considered already [40,41]. Let us assume that the thickness of each interior block is approximately the same $p_{2 k+1}-p_{2 k} \simeq \delta p$ and $\delta p$ is defined by $B$. Then, the single-particle energy in the region $L_{F C}$ can be fitted by

$$
\varepsilon(\mathbf{p})-\mu \sim \mu \frac{\delta p}{p_{F}}\left[\sin \left(\frac{p}{\delta p}\right)+b(p)\right] .
$$

The blocks are formed since all the single particle states around the minimum values of the fast sine function are occupied and those around its maximum values are empty, the average occupation being controlled by a slow function $b(\mathbf{p}) \approx \cos [\pi n(\mathbf{p})]$. It follows from Eq. (40) that the effective mass $m^{*}$ at each internal Fermi surface is of the order of the bare mass $M, m^{*} \sim M$. Upon replacing $n(\mathbf{p})$ in Eq. (5) by $\nu(\mathbf{p})$, defined by Eqs. (38) and (39), and using the Simpson's rule, we obtain that the minimum loss in the ground state energy due to formation of the blocks is about $(\delta p)^{4}$. This result can be understood by considering that the continuous $\mathrm{FC}$ function $n(\mathbf{p})$ delivers the minimum value to the energy functional $E[n(\mathbf{p})]$, while the approximation of $\nu(\mathbf{p})$ by steps of size $\delta p$ produces the minimum error of the order of $(\delta p)^{4}$. On the other hand, this loss must be compensated by the energy gain due to the magnetic field. Thus, we come to the following relation

$$
\delta p \propto \sqrt{B} .
$$

When the Zeeman splitting is taken into account in the dispersion law, Eq. (40), each of the blocks is polarized, since their outer areas are occupied only by polarized spin-up quasiparticles. The width of each areas in the momentum space $\delta p_{0}$ is given by

$$
\frac{p_{F} \delta p_{0}}{m^{*}} \sim B \mu_{e f f}
$$

where $\mu_{\text {eff }} \sim \mu_{B}$ is the effective magnetic moment of electron. We can consider such a polarization without altering the previous estimates, since it follows from Eq. (41) that $\delta p_{0} / \delta p \ll 1$. The total polarization $\Delta P$ is obtained by multiplying $\delta p_{0}$ by the number $N$ of the blocks, which is proportional to $1 / \delta p, N \sim\left(p_{f}-p_{i}\right) / \delta p$. Taking into account Eq. (41), we obtain

$$
\Delta P \sim m^{*} \frac{p_{f}-p_{i}}{\delta p} B \mu_{e f f} \propto \sqrt{B}
$$

which prevails over the contribution $\sim B$ obtained within the Landau Fermi theory. On the other hand, this quantity can be expressed as 


$$
\Delta P \propto M^{*} B
$$

where $M^{*}$ is the "average" effective mass related to the finite density of states at the Fermi level,

$$
M^{*} \sim N m^{*} \propto \frac{1}{\delta p}
$$

We can also conclude that $M^{*}$ defines the specific heat.

Eq. (41) can be discussed differently, starting with a different assumption, namely, that multiconnected Fermi sphere can be approximated by a single block. Let us put $\lambda_{0}=0$. Then, the energy gain due to the magnetic field is given by $\Delta E_{B} \sim B^{2} M^{*}$. The energy loss $\Delta E_{F C}$ due to rearrangement of the FC state can be estimated using the Landau formula [3]

$$
\Delta E_{F C}=\int(\varepsilon(\mathbf{p})-\mu) \delta n(\mathbf{p}) \frac{d \mathbf{p}^{3}}{(2 \pi)^{3}} .
$$

As we have seen above, the region occupied by the variation $\delta n(\mathbf{p})$ has the length $\delta p$, while $(\varepsilon(\mathbf{p})-\mu) \sim$ $\left(p-p_{F}\right) p_{F} / M^{*}$. As a result, we have $\Delta E_{F C}=\delta p^{2} / M^{*}$. Equating $\Delta E_{B}$ and $\Delta E_{F C}$ and taking into account Eq. (45), we arrive at the following relation

$$
\frac{\delta p^{2}}{M^{*}} \propto \delta p^{3} \propto \frac{B^{2}}{\delta p}
$$

which coincides with Eq. (41). It follows from Eqs. (43) and (44) that the effective mass $M^{*}$ diverges as

$$
M^{*} \propto \frac{1}{\sqrt{B}}
$$

Equation (48) shows that by applying a magnetic field $B$ the system can be driven back into the Landau Fermi-liquid with the effective mass $M^{*}(B)$ dependent on the magnetic field. This means that the coefficients $A(B), \gamma_{0}(B)$, and $\chi_{0}(B)$ in the resistivity, $\rho(T)=\rho_{0}+\Delta \rho$ with $\Delta \rho=A(B) T^{2}$ and $A(B) \propto\left(M^{*}\right)^{2}$, specific heat, $C / T=\gamma_{0}(B)$, and magnetic susceptibility depend on the effective mass in accordance with the Landau Fermi-liquid theory. It was demonstrated that the constancy of the well-known Kadowaki-Woods ratio, $A / \gamma_{0}^{2} \simeq$ const [42], is obeyed by systems in the highly correlated regime when the effective mass is sufficiently large [43]. Therefore, we are led to the conclusion that by applying magnetic fields the system is driven back into the Landau Fermi-liquid where the constancy of the Kadowaki-Woods ratio is obeyed. Since the resistivity is given by $\Delta \rho \propto\left(M^{*}\right)^{2}$ [43], we obtain from Eq. (48)

$$
A(B) \propto \frac{1}{B}
$$

At finite temperatures, the system remains in the Landau Fermi-liquid, but there exists a temperature $T^{*}(B)$, at which the polarized state is destroyed. To calculate the function $T^{*}(B)$, we observe that the effective mass $M^{*}$ characterizing the single particle spectrum cannot be changed at $T^{*}(B)$. In other words, at the crossover point, we have to compare the effective mass $M^{*}(T)$ defined by $T^{*}(B)$, Eq. (9), and that $M^{*}(B)$ defined by the magnetic field $B$, Eq. (48), $M^{*}(T) \sim M^{*}(B)$ 


$$
\frac{1}{M^{*}} \propto T^{*}(B) \propto \sqrt{B}
$$

As a result, we obtain

$$
T^{*}(B) \propto \sqrt{B}
$$

At temperatures $T \geq T^{*}(B)$, the system comes back into the state with $M^{*}$ defined by Eq. (9), and we observe the Landau Fermi-liquid (LFL) behavior. We can conclude that Eq. (51) determines the line in the $B-T$ phase diagram which separates the region of the $B$ dependent effective mass from the region of the $T$ dependent effective mass, see also Sec. VII. At the temperature $T^{*}(B)$, there occurs a crossover from the $T^{2}$ dependence of the resistivity to the $T$ dependence. It follows from Eq. (51), that a heavy fermion system at some temperature $T$ can be driven back into the Landau Fermi-liquid by applying a strong enough magnetic field $B \geq B_{c r} \propto\left(T^{*}(B)\right)^{2}$. We can also conclude, that at finite temperature $T$, the effective mass of a heavy fermion system is relatively field-independent at magnetic fields $B \leq B_{c r}$ and show a more pronounced metallic behavior at $B \geq B_{c r}$, since the effective mass decreases (see Eq. (48)). The same behavior of the effective mass can be observed in the Shubnikov-de Haas oscillation measurements. We note that our consideration is valid for temperatures $T \ll T_{f}$. From Eqs. (50) and (51) we obtain a unique possibility to control the essence of the strongly correlated liquid by weak magnetic fields which induce the change of the non-Fermi liquid (NFL) behavior to the LFL liquid behavior.

Now we can consider the nature of the field-induced quantum critical point in $\mathrm{YbRh}_{2} \mathrm{Si}_{2}$. The properties of this antiferromagnetic (AF) heavy fermion metal with the ordering Neèl temperature $T_{N}=70 \mathrm{mK}$ were recently investigated in Refs. [44,45]. In the AF state, this metal shows LFL behavior. As soon as the weak AF order is suppressed either by a tiny volume expansion or by temperature, pronounced deviations from the LFL behavior are observed. The experimental facts show that the spin density wave picture failed when considering the data obtained [44-46]. We assume that the electron density in $\mathrm{YbRh}_{2} \mathrm{Si}_{2}$ is close to the critical value $\left(x_{F C}-x\right) / x_{F C} \ll 1[47]$, so that the state with FC can be easily suppressed by weak magnetic fields or by the AF state. In the AF state, the effective mass is finite and the electron system of $\mathrm{YbRh}_{2} \mathrm{Si}_{2}$ possesses the LFL behavior. When the AF state is suppressed at $T>T_{N}$ the system comes back into NFL. By tuning $T_{N} \rightarrow 0$ at a critical field $B=B_{c 0}$, the itinerant AF order is suppressed and replaced by spin fluctuations [45]. Thus, we can expect the absence of any long-ranged magnetic order in this state, and the situation corresponds to a paramagnetic system with strong correlations without the field, $B=0$. As a result, the FC state is restored and we can observe NFL behavior at any temperatures in accordance with experimental facts [44]. As soon as an excessive magnetic field $B>B_{c 0}$ is applied, the system is driven back into LFL. To describe the behavior of the effective mass, we can use Eq. (48) substituting $B$ by $B-B_{c 0}$

$$
M^{*} \propto \frac{1}{\sqrt{B-B_{c 0}}} .
$$

Equation (52) demonstrates the $1 / \sqrt{B-B_{c 0}}$ divergence of the effective mass, and therefore the coefficients $\gamma_{0}(B)$ and $\chi_{0}(B)$ should have the same behavior. Meanwhile the coefficient $A(B)$ diverges as $1 /\left(B-B_{c 0}\right)$, being proportional to $\left(M^{*}\right)^{2}[43]$, and thus preserving the Kadowaki-Woods ratio, in agreement with the experimental finding [44]. To construct a $B-T$ phase diagram for $\mathrm{YbRh}_{2} \mathrm{Si}_{2}$ we use the same replacement $B \rightarrow B-B_{c 0}$ in Eq. (51) so that 


$$
T^{*}(B) \simeq c \sqrt{B-B_{c 0}}
$$

where $c$ is a constant.

The phase diagram given by Eq. (53) is in good quantitative agreement with the experimental data [44]. We note that our consideration is valid at temperatures $T \ll T_{f}$. The experimental phase diagram shows that the behavior $T^{*} \propto \sqrt{B-B_{c 0}}$ is observed up to $150 \mathrm{mK}$ [44] and allows us to estimate the magnitude of $T_{f}$, which can reach at least $1 \mathrm{~K}$ in this system. We can conclude that a new type of the quantum critical point observed in a heavy-fermion metal $\mathrm{YbRh}_{2} \mathrm{Si}_{2}$ can be identified as FCQPT with the order parameter $\kappa(\mathbf{p})$ and with the gap $\Delta_{1}$ being infinitely small $[47,48]$. Recent measurements on the heavy metal compound $\mathrm{CeRu}_{2} \mathrm{Si}_{2}$ carried out at microkelvin temperatures down to $170 \mu \mathrm{K}$ shows that the critical field $B_{c 0}$ can be as small as $0.02 \mathrm{mT}$ [49]. Note, that it follows directly from our consideration that a similar $B-T$ phase diagram given by Eq. (53) can be observed at least in the case of strongly overdoped high-temperature compounds. This is correct, except very close to the small values of both $B$ and $T$, because at $T \leq T_{c}$ the magnetic field has to be $B>B_{c}$, where $B_{c}$ is the critical field suppressing the superconductivity. We assume that this behavior was observed in overdoped Tl-2201 compounds at millikelvin temperatures [50,51].

\section{APPEARANCE OF FCQPT IN DIFFERENT FERMI LIQUIDS}

It is widely believed that unusual properties of the strongly correlated liquids observed in the hightemperature superconductors, heavy-fermion metals, 2D ${ }^{3} \mathrm{He}$ and etc., are determined by quantum phase transitions. Therefore, immediate experimental studies of relevant quantum phase transitions and of their quantum critical points are of crucial importance for understanding the physics of the high-temperature superconductivity and strongly correlated systems. In case of the high-temperature superconductors, these studies are difficult to carry out, because all the corresponding area is occupied by the superconductivity. On the other hand, recent experimental data on different Fermi liquids in the highly correlated regime at the critical point and above the point can help to illuminate both the nature of this point and the control parameter by which this point is driven. Experimental facts on strongly interacting high-density two dimensional $(2 \mathrm{D}){ }^{3} \mathrm{He}[52,53]$ show that the effective mass diverges when the density at which $2 \mathrm{D}^{3} \mathrm{He}$ liquid begins to solidify is approached [53]. Then, a sharp increase of the effective mass when the density tends to the critical density of the metal-insulator transition point, which occurs at sufficiently low densities, in a metallic 2D electron system was observed [19]. Note, that there is no ferromagnetic instability in both Fermi systems and the relevant Landau amplitude $F_{0}^{a}>-1[19,53]$, in accordance with the almost localized fermion model [54].

Now we consider the divergence of the effective mass in 2D and 3D Fermi liquids at $T=0$, when the density $x$ approaches FCQPT from the side of normal Landau Fermi liquid. First, we calculate the divergence of $M^{*}$ as a function of the difference $\left(x_{F C}-x\right)$ in case of $2 \mathrm{D}{ }^{3} \mathrm{He}$. For this purpose we use the equation for $M^{*}$ obtained in [8], where the divergence of the effective mass $M^{*}$ due to the onset of FC in different Fermi liquids including ${ }^{3} \mathrm{He}$ was predicted

$$
\frac{1}{M^{*}}=\frac{1}{M}+\frac{1}{4 \pi^{2}} \int_{-1}^{1} \int_{0}^{g_{0}} \frac{v(q(y))}{\left[1-R(q(y), \omega=0, g) \chi_{0}(q(y), \omega=0)\right]^{2}} \frac{y d y d g}{\sqrt{1-y^{2}}} .
$$

Here we adopt the notation $p_{F} \sqrt{2(1-y)}=q(y)$ with $q(y)$ being the transferred momentum, $M$ is the bare mass, $\omega$ is the frequency, $v(q)$ is the bare interaction, and the integral is taken over the coupling 
constant $g$ from zero to its real value $g_{0}$. In Eq. (54), both $\chi_{0}(q, \omega)$ and $R(q, \omega)$, being the linear response function of a noninteracting Fermi liquid and the effective interaction respectively, define the linear response function of the system in question

$$
\chi(q, \omega, g)=\frac{\chi_{0}(q, \omega)}{1-R(q, \omega, g) \chi_{0}(q, \omega)} .
$$

In the vicinity of the charge density wave instability, occurring at the density $x_{c d w}$, the singular part of the function $\chi^{-1}$ on the disordered side is of the well-known form (see e.g. [32])

$$
\chi^{-1}(q, \omega, g) \propto\left(x_{c d w}-x\right)+\left(q-q_{c}\right)^{2}+\left(g_{0}-g\right)
$$

where $q_{c} \sim 2 p_{F}$ is the wavenumber of the charge density wave order. Upon substituting Eq. (56) into Eq. (54) and integrating, the equation for the effective mass $M^{*}$ can be cast into the following form

$$
\frac{1}{M^{*}}=\frac{1}{M}-\frac{C}{\sqrt{x_{c d w}-x}}
$$

with $C$ being some positive constant. It is seen from Eq. (57) that $M^{*}$ diverges at some point $x_{F C}$ referred to as the critical point, at which FCQPT occurs as a function of the difference $\left(x_{F C}-x\right)[47]$

$$
M^{*} \propto \frac{1}{x_{F C}-x} .
$$

It follows from the derivation of Eqs. (57) and (58) that their forms are independent of the bare interaction $v(q)$. Therefore both of these equations are also applicable to $2 \mathrm{D}$ electron liquid or to another Fermi liquid. It is also seen from Eqs. (57) and (58) that FCQPT precedes the formation of charge-density waves. As a consequence of this, the effective mass diverges at high densities in case of $2 \mathrm{D}{ }^{3} \mathrm{He}$, and at low densities in case of $2 \mathrm{D}$ electron systems, in accordance with experimental facts $[19,53]$. Note, that in both cases the difference $\left(x_{F C}-x\right)$ has to be positive, because $x_{F C}$ represents the solution of Eq. (57). Thus, in considering the many-electron systems we have to replace $\left(x_{F C}-x\right)$ by $\left(x-x_{F C}\right)$. In case of a $3 \mathrm{D}$ system, the effective mass is given by [8]

$$
\frac{1}{M^{*}}=\frac{1}{M}+\frac{p_{F}}{4 \pi^{2}} \int_{-1}^{1} \int_{0}^{g_{0}} \frac{v(q(y)) y d y d g}{\left[1-R(q(y), \omega=0, g) \chi_{0}(q(y), \omega=0)\right]^{2}} .
$$

A comparison of Eq. (54) and Eq. (59) shows that there is no fundamental difference between these equations, and along the same way we again arrive at Eqs. (57) and (58). The only difference between 2D electron systems and 3D ones is that in the latter FCQPT occurs at densities which are well below those corresponding to $2 \mathrm{D}$ systems. For bulk ${ }^{3} \mathrm{He}$, FCQPT cannot probably take place since it is absorbed by the first order solidification [53].

Now we address the problem of the fermion condensation in dilute Fermi gases and in a low density neutron matter. We consider an infinitely extended system composed of Fermi particles, or atoms, interacting by an artificially constructed potential with the desirable scattering length $a$. These objects may be viewed as trapped Fermi gases, which are systems composed of Fermi atoms interacting by a potential with almost any desirable scattering length, similarly to that done for the trapped Bose gases, see e.g. [55]. If $a$ is negative the system becomes unstable at densities $x \sim|a|^{-3}$, provided the 
scattering length is the dominant parameter of the problem. That means that $|a|$ is much bigger than the radius of the interaction or any other relevant parameter of the system. The compressibility $K(x)$ vanishes at the density $x_{c 1} \sim|a|^{-3}$, making the system completely unstable [56]. Expressing the linear response function in terms of the compressibility [57],

$$
\chi(q \rightarrow 0, i \omega \rightarrow 0)=-\left(\frac{d^{2} E}{d x^{2}}\right)^{-1}
$$

we obtain that the linear response function has a pole at the origin of coordinates, $q \simeq 0, \omega \simeq 0$, at the same point $x_{c 1}$. To find the behavior of the effective mass $M^{*}$ as a function of the density, we substitute Eq. (56) into Eq. (59) taking into account that $x_{c d w}=x_{c 1}$ and $q_{c} / p_{F} \ll 1$ due to Eq. (60). At low momenta $q / p_{F} \sim 1$, the potential $v(q)$ is attractive because the scattering length is the dominant parameter and negative. Therefore, the integral on the right hand side of Eq. (59) is negative and diverges at $x \rightarrow x_{1 c}$. The above considerations can also be applied to the clarification of the fact that the effective mass $M^{*}$ is again given by Eq. (58) with $x_{F C}<x_{c 1}$. Note that the superfluid correlation cannot stop the system from squeezing, since their contribution to the ground state energy is negative. After all, the superfluid correlations can be considered as additional degrees of freedom, which can therefore only decrease the energy. We conclude that FCQPT can be observed in traps by measuring the density of states at the Fermi level, which becomes extremely large as $x \rightarrow x_{F C}$. Note that at these densities the system remains stable because $x_{F C}<x_{c 1}$. It seems quite probable that the neutron-neutron scattering length $(a \simeq-20 \mathrm{fm})$ is sufficiently large to be the dominant parameter and to permit the neutron matter to have an equilibrium energy, density, and the singular point $x_{c 1}$, at which the compressibility vanishes [58]. Therefore, we can expect that FCQPT takes place in a low density neutron matter leading to stabilization of the matter by lowering its ground state energy. A more detailed analysis of this possibility will be published elsewhere.

A few remarks are in order. We have seen that above the critical point $x_{F C}$ the effective mass $M^{*}$ is finite and, therefore, the system exhibits the Landau Fermi liquid behavior. If $\left|x-x_{F C}\right| / x_{F C} \ll 1$ the behavior can be viewed as a highly correlated one because the effective mass, being given by Eq. (58), strongly depends on the density and is very large, see Sec. VII. Beyond this region, the effective mass is approximately constant and the system becomes a normal Landau Fermi liquid. We can expect to observe such a highly correlated electron (or hole) liquid in heavily overdoped high- $\mathrm{T}_{c}$ compounds which are located beyond the superconducting dome. We recall that beyond the FCQPT point the superconducting gap $\Delta_{1}$ can be very small or even absent, see Eq. (28). Indeed, recent experimental data have shown that this liquid does exist in heavily overdoped non-superconducting $\mathrm{La}_{1.7} \mathrm{Sr}_{0.3} \mathrm{CuO}_{4}[59]$.

\section{BEHAVIOR OF HIGHLY CORRELATED LIQUID}

As we have seen in Sec. VI, when a Fermi system approaches FCQPT from the disordered phase it remains the Landau Fermi liquid with the effective mass $M^{*}$ strongly depending on the density $x_{F C}-x$, temperature and a magnetic field $B$ provided that $\left|x_{F C}-x\right| / x_{F C} \ll 1$ and $T \geq T^{*}(x)$ [47]. This state of the system, with $M^{*}$ strongly depending on $T, x$ and $B$, resembles the strongly correlated liquid. In contrast to a strongly correlated liquid, there is no the energy scale $E_{0}$ and the system under consideration is the Landau Fermi liquid at sufficiently low temperatures with the 
effective mass $M^{*} \simeq$ constant. Therefore this liquid can be called a highly correlated liquid. Obviously, a highly correlated liquid is to have uncommon properties.

In this Section, we study the behavior of a highly correlated electron liquid in magnetic fields. We show that at $T \geq T^{*}(x)$ the effective mass starts to depend on the temperature, $M^{*} \propto T^{-1 / 2}$. This $T^{-1 / 2}$ dependence of the effective mass at elevated temperatures leads to the non-Fermi liquid behavior of the resistivity, $\rho(T) \sim \rho_{0}+a T+b T^{3 / 2}$. The application of magnetic field $B$ restores the common $T^{2}$ behavior of the resistivity, $\rho \simeq \rho_{0}+A T^{2}$ with $A \propto\left(M^{*}\right)^{2}$. Both the effective mass and coefficient $A$ depend on the magnetic field, $M^{*}(B) \propto B^{-2 / 3}$ and $A \propto B^{-4 / 3}$ being approximately independent of the temperature at $T \leq T^{*}(B) \propto B^{4 / 3}$. At $T \geq T^{*}(B)$, the $T^{-1 / 2}$ dependence of the effective mass is re-established. We demonstrate that this $B-T$ phase diagram has a strong impact on the magnetoresistance (MR) of the highly correlated electron liquid. The MR as a function of the temperature exhibits a transition from the negative values of $\mathrm{MR}$ at $T \rightarrow 0$ to the positive values at $T \propto B^{4 / 3}$. Thus, at $T \geq T^{*}(B), \mathrm{MR}$ as the function of the temperature possesses a node at $T \propto B^{4 / 3}$. Such a behavior is of general form and takes place in both 3D highly correlated systems and 2D ones.

It follows from Eq. (58) that effective mass is finite provided that $\left|x-x_{F C}\right| \equiv \Delta x>0$. Therefore, the system represents the Landau Fermi liquid. In case of electronic systems the Wiedemann-Franz law is held at $T \rightarrow 0$, and Kadowaki-Woods ratio is preserved. Beyond the region $\left|x-x_{F C}\right| / x_{F C} \ll 1$, the effective mass is approximately constant and the system becomes conventional Landau Fermi liquid. On the other hand, $M^{*}$ diverges as the density $x$ tends to the critical point of FCQPT. As a result, the effective mass strongly depends on such quantities as the temperature, pressure, magnetic field given that they exceed their critical values. For example, when $T$ exceeds some temperature $T^{*}(x)$, Eq. (58) is no longer valid, and $M^{*}$ depends on the temperature as well. To evaluate this dependence, we calculate the deviation $\Delta x(T)$ generated by $T$. The temperature smoothing out the Fermi function $\theta\left(p_{F}-p\right)$ at $p_{F}$ induces the variation $p_{F} \Delta p / M^{*}(x) \sim T$, and $\Delta x(T) / x_{F C} \sim M^{*}(x) T / p_{F}^{2}$, with $p_{F}$ is the Fermi momentum and $M$ is the bare electron mass. The deviation $\Delta x$ can be expressed in terms of $M^{*}(x)$ using Eq. (58), $\Delta x / x_{F C} \sim M / M^{*}(x)$. Comparing these deviations, we find that at $T \geq T^{*}(x)$ the effective mass depends noticeably on the temperature, and the equation for $T^{*}(x)$ becomes

$$
T^{*}(x) \sim p_{F}^{2} \frac{M}{\left(M^{*}(x)\right)^{2}} \sim \varepsilon_{F}(x)\left(\frac{M}{M^{*}(x)}\right)^{2} .
$$

Here $\varepsilon_{F}(x)$ is the Fermi energy of noninteracting electrons with mass $M$. It follows from Eq. (61) that $M^{*}$ is always finite at temperatures $T>0$. We can consider $T^{*}(x)$ as the energy scale $e_{0}(x) \simeq T^{*}(x)$. This scale defines the area $\left(\mu-e_{0}(x)\right)$ in the single particle spectrum where $M^{*}$ is approximately constant, being given by $M^{*}=d \varepsilon(p) / d p$ [3]. According to Eqs. (58) and (61) it is easily verified that $e_{0}(x)$ can be written in the form

$$
e_{0}(x) \sim \varepsilon_{F}\left(\frac{x-x_{F C}}{x_{F C}}\right)^{2} .
$$

At $T \ll e_{0}(x)$ and above the critical point the effective mass $M^{*}(x)$ is finite, the energy scale $E_{0}$ given by Eq. (62) vanishes and the system exhibits the LFL behavior. At temperatures $T \geq e_{0}(x)$ the effective mass $M^{*}$ starts to depend on the temperature and the NFL behavior is observed. Thus, at $\left|x-x_{F C}\right| / x_{F C} \ll 1$ the system can be considered as a highly correlated one: at $T \ll e_{0}(x)$, the system is LFL, while at temperatures $T \geq e_{0}(x)$, the system possesses the NFL behavior.

At $T \geq T^{*}(x)$, the main contribution to $\Delta x$ comes from the temperature, therefore 


$$
M^{*} \sim M \frac{x_{F C}}{\Delta x(T)} \sim M \frac{\varepsilon_{F}}{M^{*} T}
$$

As a result, we obtaian

$$
M^{*}(T) \sim M\left(\frac{\varepsilon_{F}}{T}\right)^{1 / 2}
$$

Equation (64) allows us to evaluate the resistivity as a function of $T$. There are two terms contributing to the resistivity. Taking into account that $A \sim\left(M^{*}\right)^{2}$ and Eq. (64), we obtain the first term $\rho_{1}(T) \sim T$. The second term $\rho_{2}(T)$ is related to the quasiparticle width $\gamma$. When $M / M^{*} \ll 1$, the width $\gamma \propto\left(M^{*}\right)^{3} T^{2} / \epsilon\left(M^{*}\right) \propto T^{3 / 2}$, with $\epsilon\left(M^{*}\right) \propto\left(M^{*}\right)^{2}$ is the dielectric constant [13,43]. Combining both of the contributions, we find that the resistivity is given by

$$
\rho(T)-\rho_{0} \sim a T+b T^{3 / 2}
$$

Here $a$ and $b$ are constants. Thus, it turns out that at low temperatures, $T<T^{*}(x)$, the resistivity $\rho(T)-\rho_{0} \sim A T^{2}$. At higher temperatures, the effective mass depends on the temperature and the main contribution comes from the first term on the right hand side of Eq. (65). While $\rho(T)-\rho_{0}$ follows the $T^{3 / 2}$ dependence at elevated temperatures.

In the same way as Eq. (64) was derived, we can obtain the equation determining $M^{*}(B)[47]$. The application of magnetic field $B$ leads to a weakly polarized state, or Zeeman splitting, when some levels at the Fermi level are occupied by spin-up polarized quasiparticles. The width $\delta p=p_{F 1}-p_{F 2}$ of the area in the momentum space occupied by these quasiparticles is of the order

$$
\frac{p_{F} \delta p}{M^{*}} \sim B \mu_{e f f}
$$

Here $\mu_{\text {eff }} \sim \mu_{B}$ is the electron magnetic effective moment, $p_{F 1}$ is the Fermi momentum of the spinup electrons, and $p_{F 2}$ is the Fermi momentum of the spin-down electrons. As a result, the Zeeman splitting leads to the change $\Delta x$ in the density $x$

$$
\frac{\Delta x}{x_{F C}} \sim \frac{\delta p^{2}}{p_{F}^{2}} .
$$

We assume that $\Delta x / x_{F C} \ll 1$. Now it follows that

$$
M^{*}(B) \sim M\left(\frac{\varepsilon_{F}}{B \mu_{e f f}}\right)^{2 / 3} .
$$

We note that $M^{*}$ is determined by Eq. (66) as long as $M^{*}(B) \leq M^{*}(x)$, otherwise we have to use Eq. (58). It follows from Eq. (66) that the application of a magnetic field reduces the effective mass. Note, that if there exists an itinerant magnetic order in the system which is suppressed by magnetic field $B=B_{c 0}$, Eq. (66) has to be replaced by the equation [48], see also Sec. V,

$$
M^{*}(B) \propto\left(\frac{1}{B-B_{c 0}}\right)^{2 / 3} .
$$

The coefficient $A(B) \propto\left(M^{*}(B)\right)^{2}$ diverges as 


$$
A(B) \propto\left(\frac{1}{B-B_{c 0}}\right)^{4 / 3} .
$$

At elevated temperature, there is a temperature $T^{*}(B)$ at which $M^{*}(B) \simeq M^{*}(T)$. Comparing Eq. (64) and Eq. (67), we see that $T^{*}(B)$ is given by

$$
T^{*}(B) \propto\left(B-B_{c 0}\right)^{4 / 3} .
$$

At $T \geq T^{*}(x)$, Eq. (69) determines the line in the $B-T$ phase diagram which separates the region of the $B$ dependent effective mass from the region of the $T$ dependent effective mass. At the temperature $T^{*}(B)$, a crossover from the $T^{2}$ dependence of the resistivity to the $T$ dependence occurs: at $T<T^{*}(B)$, the effective mass is given by Eq. (67), and at $T>T^{*}(B) M^{*}$ is given by Eq. (64).

Using the $B-T$ phase diagram just presented, we consider the behavior of MR

$$
\rho_{m r}(B, T)=\frac{\rho(B, T)-\rho(0, T)}{\rho(0, T)},
$$

as a function of magnetic field $B$ and $T$. Here $\rho(B, T)$ is the resistivity measured at the magnetic field $B$ and temperature $T$. We assume that the contribution $\Delta \rho_{m r}(B)$ coming from the magnetic field $B$ can be treated within the low field approximation and given by the well-known Kohler's rule,

$$
\Delta \rho_{m r}(B) \sim B^{2} \rho\left(0, \Theta_{D}\right) / \rho(0, T),
$$

with $\Theta_{D}$ is the Debye temperature. Note, that the low field approximation implies that $\Delta \rho_{m r}(B) \ll$ $\rho(0, T) \equiv \rho(T)$. Substituting Eq. (71) into Eq. (70), we find that

$$
\rho_{m r}(B, T) \sim \frac{c\left(M^{*}(B, T)\right)^{2} T^{2}+\Delta \rho_{m r}(B)-c\left(M^{*}(0, T)\right)^{2} T^{2}}{\rho(0, T)} .
$$

Here $M^{*}(B, T)$ denotes the effective mass $M^{*}$ which now depends on both the magnetic field and the temperature, and $c$ is a constant.

Consider MR given by Eq. (72) as a function of $B$ at some temperature $T=T_{0}$. At low temperatures $T_{0} \leq T^{*}(x)$, the system behaves as common Landau Fermi liquid, and MR is an increasing function of $B$. When the temperature $T_{0}$ is sufficiently high, $T^{*}(B)<T_{0}$, and the magnetic field is small, $M^{*}(B, T)$ is given by Eq. (64). Therefore, the difference $\Delta M^{*}=\left|M^{*}(B, T)-M^{*}(0, T)\right|$ is small and the main contribution is given by $\Delta \rho_{m r}(B)$. As a result, MR is an increasing function of $B$. At elevated $B$, the difference $\Delta M^{*}$ becomes a decreasing function of $B$, and $\mathrm{MR}$ as the function of $B$ reaches its maximum value at $T^{*}(B) \sim T_{0}$. In accordance with Eq. (69), $T^{*}(B)$ determines the crossover from $T^{2}$ dependence of the resistivity to the $T$ dependence. Differentiating the function $\rho_{m r}(B, T)$ given by Eq. (72) with respect to $B$, one can verify that the derivative is negative at sufficiently large values of the magnetic field when $T^{*}(B) \simeq T_{0}$. Thus, we are led to the conclusion that the crossover manifests itself as the maximum of MR as the function of $B$.

We now consider MR as a function of $T$ at some $B_{0}$. At low temperatures $T \ll T^{*}\left(B_{0}\right)$, it follows from Eqs. (64) and (67) that $M^{*}\left(B_{0}\right) / M^{*}(T) \ll 1$, and MR is determined by the resistivity $\rho(0, T)$. Note, that $B_{0}$ has to be comparatively high to ensure the inequality, $T^{*}(x) \leq T \ll T^{*}\left(B_{0}\right)$. As a result, MR tends to $-1, \rho_{m r}\left(B_{0}, T \rightarrow 0\right) \simeq-1$. Differentiating the function $\rho_{m r}\left(B_{0}, T\right)$ with respect to $B_{0}$ we can check that its slope becomes steeper as $B_{0}$ is decreased, being proportional $\propto\left(B_{0}-B_{c 0}\right)^{-7 / 3}$. 
At $T=T_{1} \sim T^{*}\left(B_{0}\right)$, MR possesses a node because at this point the effective mass $M^{*}\left(B_{0}\right) \simeq M^{*}(T)$, and $\rho\left(B_{0}, T\right) \simeq \rho(0, T)$. Again, we can conclude that the crossover from the $T^{2}$ resistivity to the $T$ resistivity, which occurs at $T \sim T^{*}\left(B_{0}\right)$, manifests itself in the transition from negative MR to positive MR. At $T>T^{*}\left(B_{0}\right)$, the main contribution to MR comes from $\Delta \rho_{m r}\left(B_{0}\right)$, and MR reaches its maximum value. Upon using Eq. (71) and taking into account that at this point $T$ has to be determined by Eq. (69), $T \propto\left(B_{0}-B_{c 0}\right)^{4 / 3}$, we obtain that the maximum value $\rho_{m r}^{m}\left(B_{0}\right)$ of $\mathrm{MR}$ is $\rho_{m r}^{m}\left(B_{0}\right) \propto\left(B-B_{c 0}\right)^{-2 / 3}$. Thus, the maximum value is a decreasing function of $B_{0}$. At $T^{*}\left(B_{0}\right) \ll T$, MR is a decreasing function of the temperature, and at elevated temperatures MR eventually vanishes since $\Delta \rho_{m r}\left(B_{0}\right) / \rho(T) \ll 1$.

The recent paper [60] reports on measurements of the resistivity of $\mathrm{CeCoIn}_{5}$ in a magnetic field. With increasing field, the resistivity evolves from the $T$ temperature dependence to the $T^{2}$ dependence, while the field dependence of $A(B) \sim\left(M^{*}(B)\right)^{2}$ displays the critical behavior best fitted by the function, $A(B) \propto\left(B-B_{c 0}\right)^{-\alpha}$, with $\alpha \simeq 1.37$ [60]. All these facts are in a good agreement with the $B-T$ phase diagram given by Eq. (69). The critical behavior displaying $\alpha=4 / 3[47]$ and described by Eq. (68) is also in a good agreement with the data. A transition from negative MR to positive MR with increasing $T$ was also observed [60]. We believe that an additional analysis of the data [60] can reveal that the crossover from $T^{2}$ dependence of the resistivity to the $T$ dependence occurs at $T \propto\left(B-B_{c 0}\right)^{4 / 3}$. As well, this analysis could reveal supplementary peculiarities of MR. While the behavior of the heavy fermion metal $\mathrm{CeCoIn}_{5}$ in magnetic fields displayed in Ref. [60] can be identified as the highly correlated behavior of a Landau Fermi liquid approaching FCQPT from the disordered phase [47].

\section{SUMMARY AND CONCLUSION}

We have discussed the appearance of the fermion condensation, which can be compared to the BoseEinstein condensation. A number of experimental evidences have been presented that are supportive to the idea of the existence of FC in different liquids. We have demonstrated also that experimental facts collected in different materials, belonging to the high- $T_{c}$ superconductors, heavy fermion metals and strongly correlated 2D structures, can be explained within the framework of the theory based on FCQPT.

We have shown that the appearance of FC is a quantum phase transition, that separates the regions of normal and strongly correlated liquids. Beyond the fermion condensation point the quasiparticle system is divided into two subsystems, one containing normal quasiparticles, the other being occupied by fermion condensate localized at the Fermi level. In the superconducting state the quasiparticle dispersion in systems with FC can be represented by two straight lines, characterized by effective masses $M_{F C}^{*}$ and $M_{L}^{*}$, and intersecting near the binding energy $E_{0}$ which is of the order of the superconducting gap. The same quasiparticle picture and the energy scale $E_{0}$ persist in the normal state. We have demonstrated that fermion systems with FC have features of a "quantum protectorate" and shown that the theory of high temperature superconductivity, based on the fermion condensation quantum phase transition and on the conventional theory of superconductivity, permits the description of high values of $T_{c}$ and of the maximum value of the gap $\Delta_{1}$, which may be as big as $\Delta_{1} \sim 0.1 \varepsilon_{F}$ or even larger. We have also traced the transition from conventional superconductors to high- $T_{c}$ ones. We have shown by a simple, although self-consistent analysis that the general features of the shape of the critical temperature $T_{c}(x)$ as a function of the density $x$ of the mobile carriers in the high- $T_{c}$ compounds can be understood within the framework of the theory. 
We have demonstrated that strongly correlated many-electron systems with FC, which exhibit strong deviations from the Landau Fermi liquid behavior, can be driven into the Landau Fermi liquid by applying a small magnetic field $B$ at low temperatures. A re-entrance into the strongly correlated regime is observed if the magnetic field $B$ decreases to zero, while the effective mass $M^{*}$ diverges as $M^{*} \propto 1 / \sqrt{B}$. The regime is restored at some temperature $T^{*} \propto \sqrt{B}$. This behavior is of a general form and takes place in both three dimensional and two dimensional strongly correlated systems, and demonstrates the possibility to control the essence of strongly correlated electron liquids by weak magnetic fields.

The appearance of FCQPT in 2D strongly correlated structures, in trapped Fermi gases and in a low density neutron matter has been considered. We have provided an explanation of the experimental data on the divergence of the effective mass in a $2 \mathrm{D}$ electron liquid and in $2 \mathrm{D}{ }^{3} \mathrm{He}$, as well as shown that above the critical point the system exhibits the Landau Fermi liquid behavior. We expect that FCQPT takes place in trapped Fermi gases and in a low density neutron matter leading to stabilization of the matter by lowering its ground state energy. If $\left|x-x_{F C}\right| / x_{F C} \ll 1$ the behavior can be viewed as a highly correlated one because the effective mass is very large and strongly depends on the density. Beyond this region, the effective mass is approximately constant and the system becomes a normal Landau Fermi liquid.

The behavior in magnetic fields of a highly correlated electron liquid approaching FCQPT from the disordered phase has been considered. We have shown that at sufficiently high temperatures the effective mass starts to depend on $T, M^{*} \propto T^{-1 / 2}$. This $T^{-1 / 2}$ dependence of the effective mass at elevated temperatures leads to the non-Fermi liquid behavior of the resistivity. The application of a magnetic field $B$ restores the common $T^{2}$ behavior of the resistivity. We have demonstrated that this $B-T$ phase diagram has a strong impact on the magnetoresistance (MR) of the highly correlated electron liquid. The MR as a function of the temperature exhibits a transition from the negative values of $\mathrm{MR}$ at $T \rightarrow 0$ to the positive values at $T \propto B^{4 / 3}$.

We conclude that FCQPT can be viewed as a universal cause of the non-Fermi liquid behavior observed in different metals and liquids.

\section{ACKNOWLEDGMENTS}

VRS expresses his gratitude to CTSPS for the hospitality during his stay in Atlanta and for financial support that made this stay possible. MYA is grateful to the Hebrew University Intramural fund of the Hebrew University for financial support. AZM acknowledges support from the U.S. Department of Energy, Division of Chemical Sciences, Office of Basic Energy Sciences, Office of Energy Research. This work was supported in part by the Russian Foundation for Basic Research, project 01-02-17189. 


\section{REFERENCES}

[1] S.T. Belyaev, Sov. Phys. JETP, 7, 289 (1958).

[2] S.T. Belyaev, Sov. Phys. JETP, 7, 299 (1958).

[3] L. D. Landau, Zh. Éksp. Teor. Fiz. 30, 1058 (1956); [Sov. Phys. JETP 3 (1956) 920].

[4] V.A. Khodel and V.R. Shaginyan, Pis'ma Zh. Éksp. Teor. Fiz. 51, 488 (1990); [JETP Lett. 51, 553 (1990).

[5] G. E. Volovik, Pis'ma Zh. Éksp. Teor. Fiz. 53, 208 (1991); [JETP Lett. 53, 222 (1991)].

[6] M.Ya. Amusia and V.R. Shaginyan, Pis'ma Zh. Éksp. Teor. Fiz. 73, 268 (2001); [JETP Lett. 73, $232(2001)]$

M.Ya. Amusia and V.R. Shaginyan, Phys. Rev. B 63, 224507 (2001); V.R. Shaginyan, Physica B 312-313C, 413 (2002).

[7] V.A. Khodel, V.R. Shaginyan, and V.V. Khodel, Phys. Rep. 249, 1 (1994).

[8] V.A. Khodel, V.R. Shaginyan, and M.V. Zverev, Pis'ma Zh. Éksp. Teor. Fiz. 65, 242 (1997); [JETP Lett. 65, 253 (1997)].

[9] J. Dukelsky, V.A. Khodel, P. Schuck, and V.R. Shaginyan, Z. Phys. 102, 245 (1997);

V.A. Khodel and V.R. Shaginyan, Condensed Matter Theories, 12, 222 (1997).

[10] J. Bardeen, L.N. Cooper, and J.R. Schrieffer, Phys. Rev. 108, 1175 (1957).

[11] V.R. Shaginyan, Phys. Lett. A 249, 237 (1998).

[12] V.A. Khodel, J.W. Clark, and V.R. Shaginyan, Solid Stat. Comm. 96, 353 (1995).

[13] S.A. Artamonov and V.R. Shaginyan, Zh. Éksp. Teor. Fiz. 119, 331 (2001); [JETP 92, 287 (2001)].

[14] R.B. Laughlin and D. Pines, Proc. Natl. Acad. Sci. USA 97, 28 (2000).

[15] P.W. Anderson, cond-mat/0007185; cond-mat/0007287.

[16] P.V. Bogdanov et al., Phys. Rev. Lett. 85, 2581 (2000).

[17] A. Kaminski et al., Phys. Rev. Lett. 86, 1070 (2001).

[18] T. Valla et al., Science 285, 2110 (1999);

T. Valla et al., Phys. Rev. Lett. 85, 828 (2000).

[19] A.A. Shashkin, S.V. Kravchenko, V.T. Dolgopolov, and T.M. Klapwijk, Phys. Rev. B 66, 073303 (2002).

[20] G. Grüner, Density Waves in Solids (Addison-Wesley, Reading, MA, 1994).

[21] D.R. Tilley and J. Tilley, Superfluidity and Superconductivity, (Bristol, Hilger, 1985).

[22] V.R. Shaginyan, Pis'ma Zh. Éksp. Teor. Fiz. 68, 491 (1998); [JETP Lett. 68, 527 (1998)].

[23] M.Ya. Amusia and V.R. Shaginyan, Phys. Lett. A 298, 193 (2002).

[24] M. Kugler et al., Phys. Rev. Lett. 86, 4911 (2001).

[25] A.A. Abrikosov, Phys. Rev. B 52, R15738 (1995); cond-mat/9912394.

[26] M.Ya. Amusia, S.A. Artamonov, and V.R. Shaginyan, Pis'ma Zh. Éksp. Teor. Fiz. 74, 396 (1998); [JETP Lett. 74, 435 (2001)].

[27] N.-C. Yeh et al., Phys. Rev. Lett. 87087003 (2001).

[28] A. Biswas et al., Phys. Rev. Lett. 88207004 (2002).

[29] J.A. Skinta et al., Phys. Rev. Lett. 88207005 (2002).

[30] J.A. Skinta et al., Phys. Rev. Lett. 88207003 (2002).

[31] C.-T. Chen at el., Phys. Rev. Lett. 88227002 (2002).

[32] C.M. Varma, Z. Nussinov, and Wim van Saarloos, Phys. Rep. 361, 267 (2002).

[33] M.Ya. Amusia and V.R. Shaginyan, Pis'ma Zh. Éksp. Teor. Fiz. 76, 774 (2002).

[34] N. Miyakawa et al., Phys. Rev. Lett. 83, 1018 (1999). 
[35] D.L. Feng et al., cond-mat/0107073.

[36] D.S. Dessau et al., Phys. Rev. Lett. 66, 2160 (1991).

[37] A.B. Migdal, Theory of Finite Fermi Systems and Applications to Atomic Nuclei (Benjamin, Reading, MA, 1977).

[38] Z. Yusof et al., Phys. Rev. Lett. 88167006 (2002); cond-mat/01044367.

[39] S. A. Artamonov, V.R. Shaginyan, and Yu.G. Pogorelov, JETP Lett. 68, 942 (1998).

[40] M. de Llano and J. P. Vary, Phys. Rev. C 19, 1083 (1979); M. de Llano, A. Plastino, and J.G. Zabolitsky, Phys. Rev. C 20, 2418 (1979).

[41] M.V. Zverev and M. Baldo, cond-mat/9807324.

[42] K. Kadowaki and S.B. Woods, Solid State Commun. 58, 507 (1986).

[43] V.A. Khodel and P. Schuck, Z. Phys. B 104, 505 (1997).

[44] P. Gegenwart et al., Phys. Rev. Lett. 89, 056402 (2002); P. Gegenwart et al., cond-mat/0207570.

[45] K. Ishida et al., Phys. Rev. Lett. 89, 107202 (2002).

[46] P. Coleman et al., J. Phys. Condens. Matter 13, R723 (2001); P. Coleman, cond-mat/0206003.

[47] V.R. Shaginyan, cond-mat/0208568; cond-mat/0212624; cond-mat/0301019; V.R. Shaginyan, JETP Lett. in press.

[48] Yu.G. Pogorelov and V.R. Shaginyan, Pis'ma Zh. Éksp. Teor. Fiz. 76, 614 (2002).

[49] T. Takahashi et al., cond-mat/0212238.

[50] C. Proust et al., Phys. Rev. Lett. 89, 147003 (2002).

[51] A.P. Mackenzie et al., Phys. Rev. B 53, 5848 (1996).

[52] K.-D. Morhard et al., Phys. Rev. B 53, 2658 (1996).

[53] A. Casey et al., J. Low Temp. Phys. 113, 293 (1998).

[54] M. Pfitzner and P. Wölfe, Phys. Rev. B 33, 2003 (1986).

[55] S. Inouye et al., Nature, 392 (1998) 151.

[56] M.Ya. Amusia, A.Z. Msezane, and V.R. Shaginyan, Phys. Lett. A 293, 205 (2002).

[57] L.D. Landau and E.M. Lifshitz, Statistical Physics I (Adison-Wesley, Reading, MA, 1970).

[58] M.Ya. Amusia and V.R. Shaginyan, Eur. Phys. J. A 8, 77 (2000).

[59] S. Nakamae et al., cond-mat/0212283.

[60] J. Paglione et al., cond-mat/0212502. 